\title{
BMJ Open Patient and public involvement in the development of clinical practice guidelines: a scoping review protocol
}

\author{
Elizabeth Ann Bryant (D) , ${ }^{1}$ Anna Mae Scott (D) , ${ }^{2}$ Rae Thomas (id ${ }^{2}$
}

To cite: Bryant EA, Scott AM, Thomas R. Patient and public involvement in the development of clinical practice guidelines: a scoping review protocol. BMJ Open 2020;10:e037327. doi:10.1136/ bmjopen-2020-037327

- Prepublication history and additional material for this paper are available online. To view these files, please visit the journal online (http://dx.doi. org/10.1136/bmjopen-2020037327).

Received 29 January 2020 Revised 28 August 2020 Accepted 06 September 2020

Check for updates

(C) Author(s) (or their employer(s)) 2020. Re-use permitted under CC BY-NC. No commercial re-use. See rights and permissions. Published by BMJ.

${ }^{1}$ Faculty of Health Sciences and Medicine, Bond University, Gold Coast, Queensland, Australia ${ }^{2}$ Institute for Evidence-Based Healthcare, Bond University, Gold Coast, Queensland, Australia

Correspondence to Ms Elizabeth Ann Bryant; abryant@bond.edu.au

\section{ABSTRACT}

Introduction Clinical practice guidelines (CPGs) are intended to optimise patient care by recommending care pathways based on the best available research evidence and practice experience. Patient and public involvement (PPI) in healthcare is recommended based on the expectation that it will improve the quality and relevance of outcomes. There is no consensus on what constitutes meaningful and effective PPI in CPG. We will conduct a scoping review to identify and synthesise knowledge in four key areas: who have been the patients and public previously involved in CPG development, how were they recruited, at what stage in the CPG process were they involved and how were they involved. This knowledge will inform a general model of PPI in CPG to inform CPGs development.

Methods and analysis We will conduct a scoping review using the Methodology for Scoping Reviews refined by the Joanna Briggs Institute. Searches will be conducted in electronic databases (PubMed, Embase, CINAHL and PsycINF0). National standards for developing CPGs from Australia, UK, Canada and the USA will also be identified. A forward and backward citation search will be conducted on the included studies and national standards. Abstracts and full-text studies will be independently screened by two researchers. Extracted data will include study details, type of clinical guideline and the four key areas, which patients and public were involved, how were they recruited, at what stage were they included and how they were involved. Data will be narratively synthesised.

Ethics and dissemination As a scoping review, this study does not require ethics approval. We intend to disseminate the results through publication in a peer-reviewed journal and conference presentations. Furthermore, we will use the findings from our scoping review to inform future research to fill key evidence gaps identified by this review.

\section{INTRODUCTION \\ Rationale}

Clinical practice guidelines (CPGs) are intended to optimise patient care by recommending care pathways for clinicians about the management of patients with specific conditions. They should be based on the best available research evidence and practice experience. ${ }^{1}$ Patient and public involvement (PPI) in healthcare, whether in research or to inform policy decisions, is recommended

\section{Strengths and limitations of this study}

This project will follow a robust design strategy that includes an established research framework, a search strategy and a selection process.

- The search strategy includes published primary studies in different databases with peer-reviewed literature, with no restrictions on study design or date of publication.

- This study will include published national standards for developing CPGs from Australia, UK, Canada and the USA but not books or grey literature.

- We acknowledge that we may not have included relevant studies cited by the published national standards for guideline development in countries outside Australia, UK, USA and Canada.

based on ethical principles and the expectation that it will improve the relevance of the outcomes and quality of the decisions. ' 'Involvement' is characterised as an active partnership between patients and/or members of the public and others. ${ }^{1}$

International guideline standards include PPI as a core principle for developing highquality evidence-based CPGs. ${ }^{3}$ In Australia, the National Health and Medical Research Council (NHMRC) guideline standards recommend that the guideline development process should be multidisciplinary and include consumers. ${ }^{4}$ However, there is no consensus on who to include as consumers, for example, patients, caregivers, family members or consumer advocates, how many to include, at what stage in the development process to include them or how best to include them. ${ }^{156}$ However, Canada ${ }^{7}$ and the $\mathrm{UK}^{6}$ are notable exceptions as both countries have published national standards that provide some answers to those questions.

The Canadian Task Force on Preventive Health Care (CTFPHC) established to develop CPGs, published a protocol for incorporating patient experiences and views into the $\mathrm{CPG}$ development process. ${ }^{7}$ The patient engagement protocol provides guidance 
regarding who to include and when to include them. Acknowledging the limited research on PPI in guideline development, CTFPHC followed the recommendations of the Institute of Medicine (IOM) ${ }^{1}$ and the Appraisal of Guidelines for Research and Evaluation (AGREE) Collaboration. ${ }^{8}$ The IOM recommended including a patient with disease-specific experience and a patient advocate in the CPG development process. ${ }^{1}$ The AGREE Collaboration encouraged guideline developers to integrate patient experience and views into guideline development through formal consultation with patients and patient groups. ${ }^{8}$ Beginning in 2015, the CTFPHC patient engagement protocol includes an arbitrary number of patients with disease-specific experience or their caregivers at three stages of guideline development: when deciding on which screening questions the guideline should address, rating the importance of test outcomes and, usability of the knowledge translation tools, for example, patient decision aids.

Similarly, the National Institute for Health and Care Excellence (NICE) provides guidance, including clinical guidelines, for the UK National Health Service that focuses on the patient, service-user and carer. ${ }^{6}$ Their stated aim of PPI is to ensure that the experiences and perspectives of patients and public inform all NICE's guidance products. That philosophy has been maintained since 2002 and strengthened in 2013 with the publication of a policy to guide PPI in NICE guidance products. How the CTFPH and NICE established the number of public and patients to include, the methods of recruitment, when and how to include PPI in the CPG development process is not explained and limits the application of both protocols.

A recent opinion article described PPI in guideline development in terms of when to involve patients and the public, how to recruit patients and the public, how to integrate personal experience with research evidence and what models of involvement are in practice. ${ }^{9}$ The authors provided no methodology for article selection or review of articles and no articles post-2015 were included. They suggested that despite international interest in incorporating PPI as a means of improving the quality of CPGs, there are no standardised methodologies to achieve meaningful PPI in guideline development. It is not surprising then that 5 years after the $\mathrm{IOM}^{1}$ released standards for development of healthcare guidelines, only $8 \%$ of guideline developers in the USA required PPI in guideline development groups and only $20 \%$ of guideline developers in the USA created patienttargeted guideline versions. ${ }^{10}$ This is despite research literature suggesting that PPI has a positive impact on guideline development through augmenting clinical care recommendations with patient-focused issues thus helping to realise the aim of the guidelines: to optimise patient care and outcomes. ${ }^{11} 12$ For example, involving infertile couples with professionals developing a multidisciplinary guideline on infertility broadened the scope of the guideline by including patient-identified clinical issues. $^{12}$
Over a decade ago in Australia, the NHMRC suggested that a clinical guideline development panel may need to establish a separate consumer focus group to facilitate identification of all relevant consumer experiences, concerns and recommendations. ${ }^{4}$ Workshops for the members of the guideline development panels were also suggested as a way of bringing together multidisciplinary groups, including consumers, to determine the need for CPGs and discuss the methods to be used in their preparation. Evidence of this approach is scarce, but some research supports this approach as indicative of a feasible methodology for answering the PPI in guideline development questions of who, when and how. 11314

First, as part of a multidisciplinary approach to develop a CPG on early-stage chronic kidney disease, peerfacilitated workshops involving patients with chronic kidney disease and their carers were convened to identify patient-focused topics and outcomes. ${ }^{13}$ These workshops operated in parallel with the guideline working group. The recommendations of the patient/carer workshops were provided to the guideline working group. Using a before-and-after comparison of the chronic kidney disease CPGs, this approach to PPI resulted in a CPG that was patient endorsed, augmented with consumer-focused issues and complemented by a plain English decisionmaking tool. ${ }^{13}$

Second, another research group conducted parallel guideline development groups to investigate the impact of PPI on guideline question formation for diagnosing dementia. $^{14}$ One guideline group included patients, spouses, patient advocates and physicians, while the other involved physicians only. In line with previous findings, the CPG developed with PPI differed from the parallel guideline in that it included patient-relevant topics such as rate of disease progression and suggestions for dissemination of the guideline for patients and advocates. Notably, the physician-only group 'forgot' to draft a plain-language version of the questions they proposed. ${ }^{14}$ In both these approaches, PPI was limited to one or two stages of the guideline development process and those stages were arbitrarily determined by the researchers. ${ }^{13} 14$

Third, in another parallel study, guidelines for the treatment of irritable bowel syndrome were developed by guideline development groups with and without PPI. ${ }^{15}$ Both guideline development groups had standardised methods for CPG development, and for data interpretation, however, group membership differed. One guideline group comprised physicians, mental health professionals and patients, their carers and patient advocates, while the other included physicians only. Again, the CPGs developed by the multidisciplinary group were considered more patient centric and inclusive of more psychosocial concerns than those from the physician-only group.

Governments, funding bodies and guideline developers worldwide seek to involve patients and the broader public in development of CPGs. However, there is no agreed model nor consistent methodology for meaningful PPI in guideline development. We will conduct a scoping review 
to synthesise the literature that describes four key components of meaningful PPI in guideline development: who have been the patients and public previously involved in CPG, how were they recruited, at what stage in the CPG process were they involved and how were they involved.

\section{Objectives}

We aim to systematically scope the literature on PPI in the development of CPGs and to identify and synthesise knowledge about who to include, how to recruit them, at what stage in the development process to include them and the methods of involvement. We anticipate this knowledge will inform a general model that can be adopted and adapted specifically for PPI in development of CPGs.

\section{METHODS AND ANALYSIS \\ Scoping review}

A scoping review is more appropriate than a systematic review in mapping the different concepts that describe involvement of patients and public in development of CPGs. Being broader than systematic reviews, scoping reviews clarify key concepts in the literature and identify knowledge gaps. ${ }^{16}$ They are considered to be hypothesis generating rather than hypothesis testing and have the potential to inform further research directions. ${ }^{16}$ The proposed scoping review will be conducted based on the methodological framework developed by Arksey and $\mathrm{O}^{\prime}$ Malley ${ }^{17}$ and refined by the Joanna Briggs Institute (JBI). ${ }^{18}$

\section{Identifying the research question}

The primary research question has four components: who have been the patients and public previously involved in CPG, how were they recruited, at what stage in the CPG process were they involved and how were they involved. As scoping reviews are an iterative process, additional research questions may arise as we proceed. ${ }^{17}$

\section{Identifying relevant studies}

Based on preliminary searches in PubMed, a seminal article was located providing an overview of the current practices and future challenges of PPI in guideline development. ${ }^{9}$ The overview paper discussed the four questions directly related to our interest in facilitating PPI in developing CPGs, but there was no methodology provided and no articles post-2015 were reviewed. Therefore, we extracted the references directly related to the four components of our research question to assist with keyword identification. We followed the JBI's ${ }^{18}$ two-step search strategy, that is, first a limited search of PubMed, considered appropriate for this topic. The citations extracted from the overview paper $^{9}$ then served as a validation set for this stage. The search was iteratively developed, in collaboration with a research information specialist, to ensure the validation set of key papers was found in the PubMed search. The validation set was analysed for key words and phrases, and a second-step search strategy was constructed. Search terms were deliberately broad so as not to unduly limit articles. ${ }^{19}$ The search strategy constructed for PubMed is shown in online supplemental appendix 1.

The initial second-step database search was conducted on 1 October 2019 in PubMed, Embase, CINAHL and PsycINFO. Our search strategy for published studies is international and does not exclude languages other than English. Our search of the grey literature of published national standards for guideline development is restricted to Australia and three countries that have a similar social and economic environment to Australia, that is, the UK, Canada and the USA. We will analyse those published national standards separately. Additionally, we will conduct a forward and backward citation search on included studies. We anticipate that combining internationally published research and health system content from similar health environments would inform a general model that can be adopted and adapted specifically for PPI in development of Australian clinical guidelines.

\section{Study selection}

Consistent with the JBI scoping review methodology, ${ }^{18}$ a two-part study selection process will be used: (1) a title and abstract review and (2) a full-text review. In the first step, two reviewers, one of the authors, EAB and a research assistant will independently review the titles and abstracts against the inclusion/exclusion criteria. Disputes will be resolved by consensus or referring to a third reviewer if required. Potentially included full texts will be independently reviewed by the same two reviewers. All studies excluded at the full-text screening stage will be recorded with reasons for exclusion.

We will include published primary studies that report PPI in CPG development specifically addressing the questions of who have been the patients and public involved in CPG, how were they recruited, at what stage in the CPG process were they involved and how were they involved. Reference lists of reviews, reporting some or all the study questions will be examined to verify all relevant primary studies have been included. No language or date restrictions will be applied.

We will exclude letters, opinion pieces, commentaries, reports and studies focused on PPI in health technology assessments. As we are aware of a registered systematic review protocol for a review of the evaluations of PPI models, we will exclude studies on that topic. ${ }^{20}$

\section{Charting the data}

Data will be extracted from the selected articles guided by our four scoping review components. Data extraction and analysis is anticipated to be finalised in August 2020. The first author, $\mathrm{AB}$, and a research assistant have commenced piloting the data extraction form, but no data have been formally extracted. The same two researchers will extract data independently until $90 \%$ consensus is achieved. Then EAB will complete data extraction. All authors (RT, AMS and $\mathrm{EAB}$ ) will contribute to data analysis. 
Ten included studies ${ }^{21}$ will be selected at random to pilot the data extraction form that will be modified if necessary, prior to its final use. Extracted data will include: author and date of publication, study title, study location, study design, population, type of clinical guideline, that is, for screening or therapeutic purposes, and organisation/s involved in developing the guideline and outcomes.

Outcomes will include which patients and public were involved in CPG development, how were they recruited, at what stage in the CPG process were they involved and how were they involved. We will report the most frequently used strategies and any qualitative and quantitative data arising from those strategies. If available, data arising from evaluation of those strategies will be reported. The patients and public involved in the CPG development process will be described as representing one of four groups: general public: citizens/community; screening population: the affected public but without diseasespecific experience; treatment population/patients: the affected public with disease-specific experience; and advocates: representatives of interest groups and political organisations. ${ }^{22}$ Extracted data to describe recruitment of patients and public will include who made the initial contact and the method of contact, for example, email, telephone or website post. When were patients and public involved in the CPG development process will be specified, for example, guideline selection, topic or question identification, literature review, guideline writing process or draft guideline review. To adequately describe how patients and public were involved in the CPG development process, we will extract the method of involvement, for example, focus group, survey or workshop and note the level of involvement, for example, consultation or codecision.

Consistent with the scoping review methodology of $\mathrm{JBI}^{18}$ and the scoping review questions, data extracted will be described using frequency counts of populations, methods of recruitment and involvement, and stage of involvement. For all four scoping review questions, descriptions of strategies reported as successful and unsuccessful will be subject to narrative data analysis.

\section{Collating, summarising and reporting results}

Screening results will be reported using Preferred Reporting Items for Systematic Reviews and Meta-Analyses Extension for Scoping Reviews guidelines. ${ }^{23}$ Each research question will be reported separately. We will synthesise the study findings using narrative descriptions based on the themes that emerge from the extracted data.

\section{Twitter Rae Thomas @rthomasEBP}

Contributors All authors were responsible for the initial design of this study, developed the search strategy and eligibility criteria and data extraction criteria. $E A B$ executed the search strategy an commenced screening with a research assistant. EAB led the writing of manuscript. All authors contributed to and approved the final version of this protocol.

Funding The authors have not declared a specific grant for this research from any funding agency in the public, commercial or not-for-profit sectors.
Competing interests None declared.

Patient and public involvement We anticipate that the model developed for involving patients and the public in developing clinical practice guidelines through this study will be piloted in the Australian healthcare system in future studies. Neither patients nor the public have been involved in the design or the conduct of the current review. As the study is a scoping review, there are no participants.

Patient consent for publication Not required.

Ethics approval Ethics approval is not required as the study involves information that is in the public domain and freely available. The results will be disseminated through a peer-reviewed publication and presentation at conferences targeting an audience involved in clinical guideline development and patient and public involvement in healthcare.

Provenance and peer review Not commissioned; externally peer reviewed.

Open access This is an open access article distributed in accordance with the Creative Commons Attribution Non Commercial (CC BY-NC 4.0) license, which permits others to distribute, remix, adapt, build upon this work non-commercially, and license their derivative works on different terms, provided the original work is properly cited, appropriate credit is given, any changes made indicated, and the use is non-commercial. See: http://creativecommons.org/licenses/by-nc/4.0/.

\section{ORCID iDs}

Elizabeth Ann Bryant http://orcid.org/0000-0001-9569-7290

Anna Mae Scott http://orcid.org/0000-0002-0109-9001

Rae Thomas http://orcid.org/0000-0002-2165-5917

\section{REFERENCES}

1 Institute of Medicine Committee on Standards for Developing Trustworthy Clinical Practice GuidelinesGraham R, Mancher M, Miller Wolman D, et al, eds. Clinical practice guidelines we can trust. Washington DC (US: national academies press (US) Copyright 2011 by the National Academy of sciences. all rights reserved, 2011.

2 Boivin A, Richards T, Forsythe L, et al. Evaluating patient and public involvement in research. BMJ 2018;363:k5147.

3 Armstrong MJ, Rueda J-D, Gronseth GS, et al. Framework for enhancing clinical practice guidelines through continuous patient engagement. Health Expect 2017;20:3-10.

4 Council NHaMR. A quide to the development, implementation and evaluation of clinical practice guidelines. Canberra (AU: Australian Government Publishing Service, 1998. https://www.nhmrc.gov. au/about-us/publications/guide-development-evaluation-andimplementation-clinical-practice-guidelines\#block-views-block-fileattachments-content-block-1

5 Brouwers MC, Kho ME, Browman GP, et al. Agree II: advancing Guideline development, reporting and evaluation in health care. CMAJ 2010;182:E839-42.

6 National Institute for Health and Care Excellence. Developing NICE guidelines: the manual: National Institute for health and care excellence (NICE), 2015. Available: https://www.nice.org.uk/process/ pmg20/chapter/introduction-and-overview

7 Buckland D, Bashir N, Moore JE. Canadian Task force on preventive health care (CTFPHC) patient engagement protocol. Toronto (CA: Li Ka Shing Institute, St. Michael's Hospital, 2017. https:// canadiantaskforce.ca/wp-content/uploads/2017/05/CTFPHC_ Patient-Engagement_Protocol_v11_FINAL.pdf

8 TerraceL, AGREE Collaboration. Development and validation of an international appraisal instrument for assessing the quality of clinical practice guidelines: the agree project. Qual Saf Health Care 2003;12:18-23.

9 Rashid A, Thomas V, Shaw T, et al. Patient and public involvement in the development of healthcare guidance: an overview of current methods and future challenges. Patient 2017;10:277-82.

10 Armstrong MJ, Bloom JA. Patient involvement in guidelines is poor five years after Institute of medicine standards: review of guideline methodologies. Res Involv Engagem 2017;3:19-29.

11 Gregory J, Hartz-Karp J, Watson R. Using deliberative techniques to engage the community in policy development. Aust New Zealand Health Policy 2008;5:16-24.

12 Légaré F, Boivin A, van der Weijden T, et al. Patient and public involvement in clinical practice guidelines: a knowledge synthesis of existing programs. Med Decis Making 2011;31:E45-74.

13 Tong A, Lopez-Vargas $\mathrm{P}$, Howell $\mathrm{M}$, et al. Consumer involvement in topic and outcome selection in the development of clinical practice guidelines. Health Expect 2012;15:410-23. 
14 Armstrong MJ, Mullins CD, Gronseth GS, et al. Impact of patient involvement on clinical practice Guideline development: a parallel group study. Implement Sci 2018;13:55.

15 Moayyedi P, Marsiglio M, Andrews CN, et al. Patient engagement and multidisciplinary involvement has an impact on clinical Guideline development and decisions: a comparison of two irritable bowel syndrome guidelines using the same data. J Can Assoc Gastroenterol 2019;2:30-6.

16 Munn Z, Peters MDJ, Stern C, et al. Systematic review or scoping review? guidance for authors when choosing between a systematic or scoping review approach. BMC Med Res Methodol 2018;18:143.

17 Arksey H, O'Malley L. Scoping studies: towards a methodological framework. Int J Soc Res Methodol 2005;8:19-32.

18 Peters MDJ, Godfrey C, Mclnerney P, et al. Chapter 11: Scoping Reviews. In: MZ AE, ed. Joanna Briggs Institute Reviewer's Manual. The Joanna Briggs Institute, 2017.
19 Conklin A, Morris Z, Nolte E. What is the evidence base for public involvement in health-care policy?: results of a systematic scoping review. Health Expect 2015;18:153-65.

20 Bjorkqvist J, MacLennan S, MacLennan S, et al. An evaluation of existing models of patient and public involvement in the development of clinical practice guidelines: a systematic review. prospero 2018 CRD42018116712, 2018. Available: https://www.crd.york.ac.uk/ prospero/display_record.php?ID=CRD42018116712

21 Long L. Routine piloting in systematic reviews--a modified approach? Syst Rev 2014;3:77.

22 Degeling C, Carter SM, Rychetnik L. Which public and why deliberate? - a scoping review of public deliberation in public health and health policy research. Soc Sci Med 2015;131:114-21.

23 Tricco AC, Lillie E, Zarin W, et al. PRISMA extension for scoping reviews (PRISMA-ScR): checklist and explanation. Ann Intern Med 2018;169:467-73. 